Böck, Ronald; Knauf, Rainer; Sakurai, Yoshitaka ; Tsuruta, Setsuo:

Methods for path evaluation in dynamic storyboards

Zuerst erschienen in:

Proceedings // The 8th IEEE International Conference on Advanced Learning Technologies . - Los Alamitos, Calif. [u.a.] : IEEE Computer Soc., ISBN 978-0-7695-3167-0. - 2008, S. 614-616 DOI 10.1109/ICALT.2008.50 


\title{
Methods for Path Evaluation in Dynamic Storyboards
}

\author{
Ronald Böck ${ }^{1}$, Rainer Knauf ${ }^{2}$, Yoshitaka Sakurai ${ }^{3}$, Setsuo Tsuruta ${ }^{3}$ \\ ${ }^{1}$ University of Magdeburg, Germany, ronald.boeck@ovgu.de \\ ${ }^{2}$ University of Ilmenau, Germany, rainer.knauf@tu-ilmenau.de \\ ${ }^{3}$ Tokyo Denki University, Japan,ysakurai@sie.dendai.ac.jp, tsuruta@sie.dendai.ac.jp
}

\begin{abstract}
A university study is a flexible but complicated system. Therefore, many students are not able to finish their studies in the designated time. To face this problem, Tokyo Denki University introduced a Dynamic Learning Need Reflection System (DLNRS). Also, a storyboarding concept was introduced to model the network of opportunities to compose subjects towards a complete study. DLNRS supports students in scheduling a semester and storyboarding for long term career planning. Here, we introduce methods to estimate success chances for a path through a storyboard. The methods are based on AI technologies such as Data Mining and Case-Based Reasoning. By classifying the students' given path and calculating an alternative one or a supplement, if necessary, the student gets an estimation of success chances
\end{abstract}

\section{Introduction}

Modeling knowledge about processes with humans involved (like learning, e.g.) is a challenge. Moreover, the automatic processing of information and the classification of it is more complex, respectively.

The design of university curricula is such a complex task with often no flexibility. However, students need a chance to create individual curricula by their own based on their interests, wishes, and talents. In particular, at the School of Information Environment (SIE) of Tokyo Denki University an education system called DLNRS was developed and introduced.

Storyboarding [5][7] is a very general concept and complements DLNRS, which is visualized as introduced in [4] and applied in practice [1]. Moreover, the design of a study can be managed in an easy way. More concretely, we introduce an approach to evaluate curriculum plans, which were created by students [2]. The fact is that now Data Mining can be performed.

The basic idea consists of (1) the construction of a decision tree containing all storyboard paths with related success and (2) providing a success estimation for planned paths, i.e. the classification of the user given path. The nodes of a path represent the (atomic) elements of a storyboard and the leaves (called labels) denote a related success level (examination result) estimated by the degree of success afforded by former students. To classify a path, the tree is traversed. The success is computed as the weighted average success level. Additionally, [2] introduces a technology to suggest a possible modification of a no-classified curriculum leading to an optimum.

\section{Storyboarding}

A storyboard, based on [5] and [7], is a semi-formal representation of process knowledge with human involved. The concept enjoys (1) clarity by providing a high-level modeling approach by using different degrees of granularity, (2) simplicity, and (3) visual appearance. A storyboard is defined as follows:

- A storyboard is a nested hierarchy of directed graphs with annotated, attributed nodes and edges.

- Nodes are episodes or scenes.

- Graphs are interpreted by paths through them.

- Episodes denote a sub-graph.

- Scenes denote non-decomposable leaves in the graph hierarchy and represent a learning activity. At and below the scene level is no formalism.

- The start node of a (sub-) graph is the starting point of a legal graph traversing. The end node is the corresponding target point.

- Edges (colored) denote transitions between nodes.

Figure 1 shows a small part of a possible academic curricula storyboard. For detailed information, see [4], [5] and [7]. Examples are shown in [1] and [2].

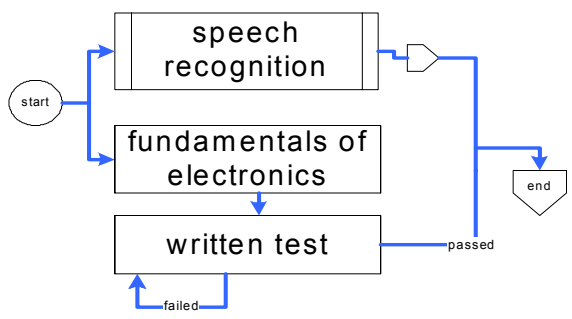

Figure 1: An example of a curricula 


\section{Storyboarding becomes a model}

For formally modeling, we distinguish between (1) basic sets of storyboard elements and (2) alphabets derived from these to model storyboard paths. Let

- $\sigma=\left\{s_{l}, \ldots, s_{n}\right\}(n \in N)$ be the set of the scenes in a storyboard throughout all its graph levels,

- $\kappa=\{$ start, end $\}$ be the set with start/end nodes, and

- $\Gamma=\sigma \cup \kappa$ be the complete alphabet of all nondecomposable storyboard elements.

Here, a method to re-name nodes is applied, because different nodes may have the same label, if they are situated in different graphs. So, all nodes need a unique label. For this purpose, referencing is introduced, e.g. st.start. Thus, every node can clearly be identified.

Finally, a path $\gamma$ is a word composed by elements of the storyboard-alphabet $\Gamma$, i.e. $\gamma=a_{1} \circ \ldots \circ a_{n}$.

\section{Decomposition}

Storyboards consist of decomposable (episodes) and non-decomposable elements (scenes). For calculation of the storyboard-tree we need atomic nodes only. So, we decompose the given paths through replacing all episodes by their corresponding sub-graphs throughout all hierarchy levels, recursively.

For example, if $\gamma=s_{1} \circ e_{1} \circ s_{2} \circ \ldots \circ s_{n}$ is a given path and let $s_{27} \circ s_{35}$ be the sub-graph of episode $e_{1}$, which is replaced. It is decomposed to $\gamma=\mathrm{s}_{1} \circ e_{1}$.start $\circ e_{1} . \mathrm{s}_{27} \circ e_{1} \cdot \mathrm{s}_{35} \circ e_{1}$.end $\circ \mathrm{s}_{2} \circ \ldots \circ \mathrm{s}_{\mathrm{n}}$. Also, we distinguish sequential from parallel structures. Sequentials form a sub-path; parallels form a single element handled as one path element.

\section{Construction of the storyboard-tree}

Based on the (decomposed) paths (derived from alphabet $\Gamma$ ) we will construct a decision tree that we call storyboard-tree. To construct such a tree we need a metrics that allows for a new path (1) merging it into a storyboard-tree when constructing and (2) finding a most similar path in the storyboard-tree when utilizing it. A metric that reflects the application is a so called least common denominator (l.c.d.), which is the longest common start sequence of the "new" path and the most similar path, which is already in the tree, starting by the root. The result is the last node $a_{i}$ both paths have in common. This never fails because a legal paths need to start with the top level start node called st.start in [2].

Here, we need a complex evaluation system. We introduced a pseudo-numeric class [2] that models both binary and real numeric cases. The evaluation marks only need to meet the requests of (1) being a finite set with (2) an ordering relation in-between. Since educational evaluation systems meet these requirements, this concept is universal. An evaluation label (referring to a complete path) contains:

- the classification result (often as an interval of formerly achieved evaluation classes),

- classifications details, i.e. a list of classes $k$ along with their respective cardinalities $h_{k}$, and

- the weighted arithmetic average: ${ }_{W A A}=\frac{\sum_{k=1}^{n} k h_{k}}{\sum_{k=1}^{n} h_{k}}$

Initially, the tree is empty and the first path is inserted easily. The other paths are inserted as follows:

- Beginning at the root, the tree and the path to be merged into are traversed coeval as long as there is a sub-tree root equal to the path's next element

- Let $a_{i}$ be the node, at which the traversing is stopped. Beginning at $a_{i}$, the remaining path is inserted as a new sub-tree.

- Finally, the corresponding label is attached as the leaf of this sub-tree. If the path is shorter it is inserted at an inner node. If the path is fully represented in the tree, nothing is extended; only the label elements are actualized.

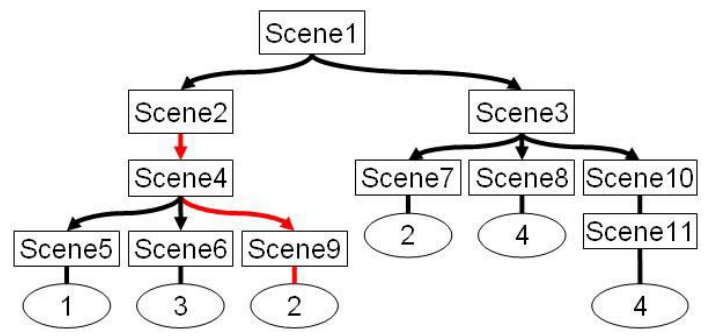

Figure 2: An example storyboard tree

Figure 2 shows a small example of a storyboardtree. For simplicity, the labels (elliptic nodes) are reduced to the WAA. According to the storyboard the nodes are represented rectangles. A path through the tree is defined by its directed edges. In [2], a prototypical implementation is given.

\section{Classification of a path 6.1 Classification process}

Estimating success chances is a classification issue. For this, the tree is traversed in a depth-first manner until a node of the path is different from all possible successor nodes of the tree's current node.

Two cases need to be distinguished, (1) the submitted path is full represented or (2) it is not. In the first case the classification result is presented. 
The second case is a more complex:

1. Let $r$ be the remaining length, i.e. the number nodes behind the $r_{i}$-th node $a_{i}$ in the path with a length $n: r=n-r_{i}$.

2. The degree of similarity $g_{p}$ between the new given path and the (according to l.c.d.) most similar one in the tree is estimated by $g_{p}=r_{i} / n$.

3. Also the $W A A$-value of the submitted path is established by merging all labels in the sub-trees under the root $a_{i}$ and visualized.

\subsection{Constructing an optimal supplement path}

After calculating the l.c.d. we have the node $a_{i}$ specified above, which is the starting point of the subtree. In this, a breadth-first search for the sub-path leading to the numeric best label is started. Here, the $W A A$ is used, but other metrics are possible.

The special quality is that the path is visualized in a re-composed way (counterpart of decomposition), performed recursively in a bottom-up manner.

Using the information of the alternative path the user can modify his with respect to the results. Surely, he can keep with the risk that the result is not the best.

Figure 2 shows an supplement (red edges), which is presented, where Scene 2 is the l.c.d. result and the starting node $(W A A$-value $=2)$. The alternative consists of the starting sequence and the recommendation.

For prototypical Prolog implementations, see [2].

\section{Conclusion and outlook}

DLNRS at the SIE provides an application and excellent test bed for storyboarding. Here, it helps the students to create their curricula for studies by their own and makes the planning process in whole easier.

The introduced methods help students by utilizing experiences of former ones with similar curricula. Based on the classified path of alumni, a storyboardtree is built. Freshmen can classify their curricula and estimate its success chance.

Current work is focused to the following issues.

A PHP implementation is also desirable. [6] provides a firm basis for this work.

A sufficient number of data sets from practice is essential to derive a reliable success estimation. Therefore, we are in the process of data collection, especially in cooperation with SIE.

Our upcoming work is directed towards solving the following issues.

A definition of (formally to check) criteria allowing the specification of individual goal-driven storyboards. It depends on cultures, countries, and universities. Thus, we plan to do that prototypically for SIE.
Storyboards have also a high performance with respect of planning. However, there is (still) no capability to manage these processes according to resources (e.g. to concretely planning timetables with several restrictions). We expect a synergy effect by incorporating the capabilities of the DLNRS.

Including meta-knowledge is another focus to infer learning needs, desires, preferences and talents. This knowledge is useful for maintaining the university's educational resources according to the needs through prediction about upcoming students' learning needs.

Individual learning plans shouldn't only be based on individual quantitative capability, but also on individual properties, talents and preferences. Consequently, we need to include some sort of user profile to avoid lavishing the students.

\section{References}

[1] Böck, R., 2006. Storyboarding (in German), student research project, University of Ilmenau, Faculty of Computer Science and Automation, Chair of Artificial Intelligence.

[2] Böck, R., 2007. Ein Data Mining Verfahren zur Pfadbewertung in Storyboards (German), Diploma Thesis, University of Ilmenau.

[3] Dohi, S., Nakamura, S., 2003. The development of the Dynamic Syllabus for School of Information Environment, ITHET03, pp. 505-510.

[4] Dohi, S., Sakurai, Y., Tsuruata, S., Knauf, R., 2006. Managing Academic Education through Dynamic Storyboarding, Proc. World Conf. on e-Learning in Corporate, Government, Healthcare, \& Higher Education 2006 (E-LEARN 2006), Honolulu (HI), USA, Chesapeake, VA: AACE, ISBN 1-880094-60-6, pp. 1611-1619.

[5] Jantke, K.P., Knauf, R., 2005. Didactic design through storyboarding: Standard concepts for standard tools, Proc. $4^{\text {th }}$ Internat. Symposium on Information and Communication Technologies, Workshop on Dissemination of e-Learning Technologies and Applications, Cape Town, South Africa, 2005, N.Y.: ACM Press, ISBN 0-9544145-6-X, pp. 20 - 25.

[6] Kasperski, S., 2007. Entwicklung einer unabhängigen Storyboardrepräsentation. (German), student research project, University of Ilmenau, Faculty of Computer Science and Automation, Germany, 2007.

[7] Knauf, R., Jantke, K.P., 2006. Storyboarding - An AI technology to represent, process, evaluate, and refine didactic knowledge, Jantke \& Kreuzberger (eds.): Knowledge Media Technologies, Proc. of the First Core-to-Core Workshop, Dagstuhl Castle, Germany, 2006, TU Ilmenau, ISSN 16179048, pp. $170-179$. 